\title{
Hall Effect in a Quasi-One-Dimensional System.
}

\author{
A.V. Lopatin \\ Department of Physics, Rutgers University, Piscataway, NJ 08855
}

(November 18, 2017)

\begin{abstract}
We consider the Hall effect in a system of weakly coupled one-dimensional chains with Luttinger interaction within each chain. We construct a perturbation theory in the inter-chain hopping term and find that there is a power law dependence of the Hall conductivity on the magnetic field with an exponent depending on the interaction constant. We show that this perturbation theory becomes valid if the magnetic field is sufficiently large.
\end{abstract}

\section{INTRODUCTION}

It is well known that one-dimensional interacting electrons form a non-Fermi-liquid system. The Green function acquires an anomalous scaling power $2 \Delta$ which implies the breakdown of the basic assumption of the Fermy-liquid theory. However, not all correlation functions have anomalous scaling: the correlators containing only density and current operators are similar to those of a noninteracting system. Therefore most of physical quantities are usual and it is interesting to consider those which do have a nontrivial contribution from the interaction.

In this paper we consider the influence of the electron interaction on the Hall effect in a quasi-one-dimensional system. To understand the effect of two-dimensionality let us consider a system of weakly coupled one-dimensional chains as an example of a quasi-onedimensional system. The inter-chain hopping term $t_{\perp}$ can be neglected for electrons with 
sufficiently large energy. Therefore the system behaves as one-dimensional on scales much smaller than $l \sim \frac{\epsilon_{F}}{t_{\perp}} a_{x}$, where $a_{x}$ is the distance between the atoms within the chains and $\epsilon_{F}$ is the Fermy energy. Actually, the anomalous scaling of Green functions leads to a correction of the above qualitative expression to $l \sim\left(\frac{t_{\perp}}{\epsilon_{F}}\right)^{\frac{-1}{1-2 \Delta}} a_{x}$ (see 5 , 9 ). On scales larger than $l$ the system is essentially two-dimensional and one-dimensional Green functions do not give even qualitatively right answer. Therefore if a physical effect comes from the length scales smaller than $l$, then the answer can still have one-dimensional anomalies. We show that the Hall effect in this system is strongly affected by the anomalous powers and acquires a power law dependence on the magnetic field

$$
\sigma_{\perp} \sim H^{-1+4 \Delta}
$$

This result is valid only if the magnetic field is strong enough. Indeed, the Hall effect is due to interference on scales $l_{H} \sim \frac{\Phi_{0}}{a_{y} H}$, where $\Phi_{0}$ is a quantum of magnetic flux and $a_{y}$ is the interchain distance. We want the system to be "one dimensional" on these scales, i.e. we need $l \gg l_{H}$. Substituting the expression for $l$ we get

$$
\frac{H a_{x} a_{y}}{\Phi_{0}} \gg\left(\frac{t_{\perp}}{\epsilon_{F}}\right)^{\frac{1}{1-2 \Delta}} .
$$

To find the Hall conductivity we use perturbation theory in $t_{\perp}$. This perturbation theory becomes valid when the condition (11) is satisfied because in this case the effect of hopping term is small on the scales important for the Hall effect.

The main technical difficulty of this problem will be that the Hall conductivity of the chains with the linear electron spectrum

$$
\epsilon_{ \pm}= \pm v_{F}\left(p \mp p_{F}\right)
$$

is zero due to the particle-hole symmetry. Therefore it is necessary to consider a nonlinear correction to the spectrum

$$
\epsilon_{ \pm}= \pm v_{F}\left(p \mp p_{F}\right)+\alpha\left(p \mp p_{F}\right)^{2}
$$

We consider the simplest model of spinless electrons. It is also assumed that the system is not close to half filling so that the umklapp processes can be neglected. 
So the Hamiltonian of the problem is

$$
\begin{array}{r}
H=v_{F} \int d x \sum_{i} \hat{\psi}_{i}^{\dagger} \tau_{3}\left(-i \partial_{x}\right) \hat{\psi}_{i}-\alpha \int d x \sum_{i} \hat{\psi}_{i}^{\dagger} \partial_{x}^{2} \hat{\psi}_{i} \\
+g \int d x \sum_{i} \hat{\psi}_{i+}^{\dagger} \hat{\psi}_{i+} \hat{\psi}_{i-}^{\dagger} \hat{\psi}_{i-}+t_{\perp} \int d x \sum_{<i, j>} \hat{\psi}_{i}^{\dagger} \hat{\psi}_{j} e^{-i \frac{e}{c} A_{i, j}}
\end{array}
$$

where $\hat{\psi}$ is composed from the right- and left-moving electrons $\hat{\psi}=\left(\begin{array}{c}\hat{\psi}_{+} \\ \hat{\psi}_{-}\end{array}\right), \tau_{3}$ is a Pauli matrix, $A_{i, j}=\int_{i}^{j} \mathbf{A} d \mathbf{l}$ and we use the Landau gauge $A_{y}=H x$. The second term in the Hamiltonian is the nonlinear correction to the free electron spectrum. The model without the hopping term and $\alpha$-term can be solved exactly, for example by the bosonization method. It is also possible to bosonize the $\alpha$-term $⿴$ but it leads to a cubic interaction between the bosons and the model with such an interaction is not exactly solvable. Therefore we have to consider the $\alpha$-term as a perturbation, too. The nonlinear term in the spectrum (2) is small compared with the linear term on scales bigger than $\frac{\alpha}{v_{F}} \sim a$, hence a perturbation theory in $\alpha$ is valid if $l_{H} \gg a$. This condition can be written as

$$
\frac{H a_{x} a_{y}}{\Phi_{0}} \ll 1
$$

and it is satisfied for any real experimental situation. Therefore a perturbation theory in $\alpha$ is always a good approximation.

The plan of the paper is the following: In Section II we express the Hall conductivity through the single-chain correlation functions. In Section III we explain the technique that we will use to calculate one-dimensional correlators. In Section IV we calculate the Hall conductivity. Finally, we summarize our results and discuss their possible applications in Section丩.

\section{EXPRESSION FOR THE HALL CONDUCTIVITY .}

Let us choose a coordinate frame so that the magnetic field points along the z-axis and the chains are along the x-axis. The label $i$, which denotes the number of the chain, increases 
in the y-direction. The electric field $E_{x}=E_{0} e^{-i \omega t}$ is applied along the chains (x-axis). In this geometry the Hall conductivity $\sigma_{\perp}(\omega)$ relates the electric field $E_{x}$ with the current between the chains $j_{y}$

$$
j_{y}=\sigma_{\perp}(\omega) E_{x}
$$

According to the Kubo formula the conductivity is expressed through the retarded currentcurrent correlator

$$
\sigma_{\perp}(\omega)=\frac{1}{\omega} \sum_{i} \int d x P_{R}(x, i, \omega)
$$

where

$$
P_{R}(x, i, \omega)=\int_{-\infty}^{\infty} d t e^{i \omega t}\left[\hat{j}_{y}(x, i, t), \hat{j}_{x}(0,0,0)\right] \theta(t)
$$

In these expressions $\hat{j}_{y}$ is the Heisenberg operator of the current between the chains

$$
\hat{j}_{y}(x, i, 0)=t_{\perp} e i\left(\hat{\psi}_{i}^{\dagger}(x) \hat{\psi}_{i+1}(x) e^{-i \frac{e}{c} A_{i, i+1}}-h . c .\right)
$$

and $\hat{j}_{x}$ is the in-chain current operator

$$
\hat{j}_{x}(x, i, 0)=e\left(v_{F} \hat{\psi}_{i}^{\dagger}(x) \tau_{3} \hat{\psi}_{i}(x)+2 \alpha \hat{\psi}_{i}^{\dagger}(x)\left(-i \partial_{x}\right) \hat{\psi}_{i}(x)\right)
$$

We will work in the Euclidean space which corresponds to the Wick rotation $t \rightarrow-i t$, $\omega \rightarrow i \omega$ (See for exampld). The Euclidean Lagrangian of this problem is

$$
\begin{array}{r}
L_{\mathcal{E}}=\int d x \int d t \sum_{i} \psi_{i}^{\dagger}\left(-\partial_{0}+i v_{F} \tau_{3} \partial_{1}+\alpha \partial_{1}^{2}\right) \psi_{i}-g \int d x \int d t \sum_{i} \psi_{i+}^{\dagger} \psi_{i+} \psi_{i-}^{\dagger} \psi_{i-} \\
-t_{\perp} \int d x \int d t \sum_{<i, j>} \psi_{i}^{\dagger} \psi_{j} e^{-i \frac{e}{c} A_{i, j}}
\end{array}
$$

where $\psi^{\dagger}, \psi$ are two-vector anticommuting variables which correspond to the operators $\hat{\psi}^{\dagger}, \hat{\psi}$ of the Hamiltonian formalism and $\partial_{0}=\frac{\partial}{\partial t}, \partial_{1}=\frac{\partial}{\partial x}$. Let us define the Euclidean currentcurrent correlator

$$
P_{\mathcal{E}}(x, i, \omega)=\int_{-\infty}^{\infty} d t e^{i \omega t}\left\langle T_{t} \hat{j}_{y}(x, i, t), \hat{j}_{x}(0,0,0)\right\rangle
$$


where $T_{t}$ means time ordering in the "Euclidean" time. For Euclidean correlators the standard perturbation theory can be used. The retarded correlator (5) is the analytical continuation of the Euclidean one

$$
P_{R}(\omega)=-i P_{\mathcal{E}}(-i \omega)
$$

Considering the last term in (8) as a perturbation, one can find the Euclidean currentcurrent correlator to the first order in $t_{\perp}$

$$
\begin{array}{r}
\int d x_{3} \sum_{i_{3}}\left\langle j_{x}\left(x_{3}, i_{3}, t_{3}\right) j_{y}\left(x_{1}, i_{1}, t_{1}\right)\right\rangle_{L_{\mathcal{E}}} \\
=2 e t_{\perp}^{2} \sum_{s} \int d x_{3} \int d x_{2} \int d t_{2}\left[\left\langle j\left(x_{3}, t_{3}\right) \psi_{s}\left(x_{1}, t_{1}\right) \psi_{s}^{\dagger}\left(x_{2}, t_{2}\right)\right\rangle\left\langle\psi_{s}\left(x_{2}, t_{2}\right) \psi_{s}^{\dagger}\left(x_{1}, t_{1}\right)\right\rangle\right. \\
\left.+\left(x_{1}, t_{1} \leftrightarrow x_{2}, t_{2}\right)\right] \sin q\left(x_{1}-x_{2}\right)
\end{array}
$$

where $q=\frac{e H a_{y}}{c}$ and the label $s=+,-$. Note that the one-dimensional current $j$ contains a contribution from the nonlinear correction to the spectrum

$$
j=j^{(0)}+j^{(1)}
$$

where

$$
\begin{array}{r}
j^{(0)}=e v_{F} \psi^{\dagger} \tau_{3} \psi \\
j^{(1)}=2 e \alpha \psi^{\dagger}\left(-i \partial_{1}\right) \psi
\end{array}
$$

The angular brackets on the r.h.s. of (11) represent averaging with respect to the single-chain Lagrangian

$$
L_{\mathcal{E}}^{(1)}=\int d x \int d t\left(\psi^{\dagger}\left(-\partial_{0}+i v_{F} \tau_{3} \partial_{1}+\alpha \partial_{1}^{2}\right) \psi-g \psi_{+}^{\dagger} \psi_{+} \psi_{-}^{\dagger} \psi_{-}\right)
$$

Finally for the Hall conductivity we have the following expression

$$
\sigma_{\perp}(\omega)=-\frac{2 e i t_{\perp}^{2}}{\omega} \Gamma_{\mathcal{E}}(-i \omega)
$$

where $\Gamma_{\mathcal{E}}(\omega)$ is the Euclidean correlator

$$
\Gamma_{\mathcal{E}}(\omega)=\int_{-\infty}^{\infty} d t e^{i \omega t} \Gamma_{\mathcal{E}}(t)
$$


with

$$
\begin{array}{r}
\Gamma_{\mathcal{E}}\left(t_{1}-t_{3}\right) \\
=\sum_{s} \int d x_{3} \int d x_{2} \int d t_{2}\left[\left\langle j\left(x_{3}, t_{3}\right) \psi_{s}\left(x_{1}, t_{1}\right) \psi_{s}^{\dagger}\left(x_{2}, t_{2}\right)\right\rangle\left\langle\psi_{s}\left(x_{2}, t_{2}\right) \psi_{s}^{\dagger}\left(x_{1}, t_{1}\right)\right\rangle\right. \\
\left.+\left(x_{1}, t_{1} \leftrightarrow x_{2}, t_{2}\right)\right] \sin q\left(x_{1}-x_{2}\right) .
\end{array}
$$

\section{BOSONIZATION.}

Now the problem is to find the one-dimensional correlators in (17). The standard way to treat a one-dimensional model with the linear spectrum is bosonization. Without the $\alpha$-term the model (15) can be solved exactly. We will treat this term as a perturbation and begin with bosonization of the Hamiltonian. The $\alpha$-term in the fermionic Lagrangian

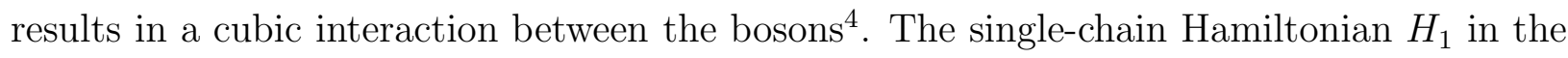
bosonized form (see Appendix A) is

$$
\hat{H}_{1}=\frac{1}{2}\left(\left(\partial_{1} \hat{\Phi}\right)^{2}+\hat{\Pi}^{2}\right)+\frac{\alpha}{3} \beta \partial_{1} \hat{\Phi}\left(3 \frac{\pi}{\beta^{2}} \hat{\Pi}^{2}+\frac{\beta^{2}}{\pi}\left(\partial_{1} \hat{\Phi}\right)^{2}\right)
$$

where $\Phi$ and $\Pi$ are canonically conjugate Bose-operators and $\beta$ is a constant which depends on the interaction constant $g$

$$
\beta=\sqrt{\pi}\left(\frac{v_{F}-g / 2 \pi}{v_{F}+g / 2 \pi}\right)^{\frac{1}{4}}
$$

The fermionic and current operators (see Appendix A) are

$$
\begin{array}{r}
\hat{\psi}_{ \pm}(x, t)=\frac{1}{\sqrt{2 \pi \delta}} e^{ \pm i \hat{\Phi}_{ \pm}(x, t)}, \quad \hat{\Phi}_{ \pm}(x, t)=\beta \hat{\Phi}(x, t) \mp \frac{\pi}{\beta} \int_{-\infty}^{x} d x^{\prime} \hat{\Pi}\left(x^{\prime}, t\right) \\
\hat{j}=-e\left(\frac{v_{F}}{\beta} \hat{\Pi}+2 \alpha \hat{\Pi} \partial_{1} \hat{\Phi}\right)
\end{array}
$$

where $\delta$ is the inverse momentum-space cutoff, and the normal ordering of operators is implied in (18) and (20). Here we have rescaled the energy units so that the velocity of the Bose-particles $v_{B}$ is 1 . The second term in the current operator is due to the $\alpha$-term in the Hamiltonian. It is more convenient to calculate the correlation functions using the 
functional representation. In this formalism we introduce the fields $\Phi, \Pi$ which are related to the fermion fields $\psi^{\dagger}, \psi$ via

$$
\begin{aligned}
& \psi_{ \pm}(x, t)=\frac{1}{\sqrt{2 \pi \delta}} e^{ \pm i \Phi_{ \pm}(x, t)}, \quad \Phi_{ \pm}(x, t)=\beta \Phi(x, t) \mp \frac{\pi}{\beta} \int_{-\infty}^{x} d x^{\prime} \Pi\left(x^{\prime}, t\right) \\
& j=-e\left(\frac{v_{F}}{\beta} \Pi+2 \alpha \Pi \partial_{1} \Phi\right) .
\end{aligned}
$$

The Green function

$$
G\left(x_{1}-x_{2}, t_{1}-t_{2}\right)=\left\langle T_{t} \hat{\psi}\left(x_{1}, t_{1}\right) \hat{\psi}^{\dagger}\left(x_{2}, t_{2}\right)\right\rangle_{H}
$$

calculated by the Hamiltonian method is related to the Green function

$$
G_{f}\left(x_{1}-x_{2}, t_{1}-t_{2}\right)=\left\langle\psi\left(x_{1}, t_{1}\right) \psi^{*}\left(x_{2}, t_{2}\right)\right\rangle_{f},
$$

calculated by the functional method through the following relation

$$
G\left(x_{1}-x_{2}, t_{1}-t_{2}\right)=\operatorname{sgn}\left(t_{1}-t_{2}\right) G_{f}\left(x_{1}-x_{2}, t_{1}-t_{2}\right) .
$$

Indeed, due to the fundamental property of the correspondence between the Hamiltonian and functional methods we have

$$
\left\langle T_{t}^{(b o z)} \hat{\psi}\left(x_{1}, t_{1}\right) \hat{\psi}^{\dagger}\left(x_{2}, t_{2}\right)\right\rangle_{H}=\left\langle\psi\left(x_{1}, t_{1}\right) \psi^{*}\left(x_{2}, t_{2}\right)\right\rangle_{f}
$$

where $T_{t}^{(b o z)}$ means time-ordering of the boson operators because the $\hat{\psi}$-operators are constructed from the boson operators. But the definition (23) implies time-ordering of fermions, and this difference should be corrected by inserting $\operatorname{sgn}\left(t_{1}-t_{2}\right)$ into (24). So to get an actual "Hamiltonian" Green function we should multiply the corresponding "functional" Green function by $\operatorname{sgn}\left(t_{1}-t_{2}\right)$.

The Lagrangian density corresponding to the single-chain Hamiltonian (18) in the Euclidean space is

$$
\mathcal{L}_{\mathcal{E}}^{(1)}=\mathcal{L}_{\mathcal{E}}^{(0)}+V\left(\Pi, \partial_{1} \Phi\right)
$$

where 


$$
\begin{array}{r}
\mathcal{L}_{\mathcal{E}}^{(0)}=i \Pi \partial_{0} \Phi-\frac{1}{2}\left(\Pi^{2}+\left(\partial_{1} \Phi\right)^{2}\right), \\
V\left(\Pi, \partial_{1} \Phi\right)=-\frac{\alpha}{3} \beta \partial_{1} \Phi\left(3 \frac{\pi}{\beta^{2}} \Pi^{2}+\frac{\beta^{2}}{\pi}\left(\partial_{1} \phi\right)^{2}\right) .
\end{array}
$$

We will treat the interaction $V$ as a perturbation. The bare correlation functions are

$$
\begin{array}{r}
\left\langle\Phi\left(x_{1}, t_{1}\right) \Phi\left(x_{2}, t_{2}\right)\right\rangle_{f}^{(0)}=-d(x, t) \\
\int_{-\infty}^{x_{1}} d x^{\prime} \int_{-\infty}^{x_{2}} d x^{\prime \prime}\left\langle\Pi\left(x^{\prime}, t_{1}\right) \Pi\left(x^{\prime \prime}, t_{2}\right)\right\rangle_{f}^{(0)}=-d(x, t) \\
\int_{-\infty}^{x_{1}} d x^{\prime}\left\langle\Pi\left(x^{\prime}, t_{1}\right) \Phi\left(x_{2}, t_{2}\right)\right\rangle_{f}^{(0)}=-\frac{i}{2 \pi} \operatorname{sgn} t\left(\arctan \frac{x}{|t|+\delta}+\frac{\pi}{2}\right),
\end{array}
$$

where

$$
d(x, t)=\frac{1}{4 \pi} \ln \frac{(|t|+\delta)^{2}+x^{2}}{l^{2}} .
$$

In the above formulas $t=t_{1}-t_{2}$ and $x=x_{1}-x_{2}$. The label (0) in (28-30) means averaging with respect to the free-boson Lagrangian $L_{\mathcal{E}}^{(0)}$ and $l$ is the length of the chains. Using the correlators (28) we can find the correlators of $\Phi_{ \pm}$:

$$
\begin{array}{r}
\left\langle\Phi_{ \pm}\left(x_{1}, t_{1}\right) \Phi_{ \pm}\left(x_{2}, t_{2}\right)\right\rangle^{(0)}-\left\langle\Phi_{ \pm}(0,0) \Phi_{ \pm}(0,0)\right\rangle^{(0)} \\
=-\frac{1}{4}\left(\frac{\pi}{\beta^{2}}+\frac{\beta^{2}}{\pi}\right) \ln \frac{t^{2}+x^{2}}{\delta^{2}} \pm i \arctan \frac{x}{t}
\end{array}
$$

Here and below we put $\delta=0$ wherever it does not lead to apparent divergences.

Now let us reproduce the well known result for the one-particle Green function. According to the formula

$$
\left\langle e^{A}\right\rangle=e^{\frac{1}{2}\left\langle A^{2}\right\rangle}
$$

where $A$ can be any linear functional of the fields $\Pi, \Phi$, we can easily find the correlators of $\psi$-functionals

$$
G_{ \pm, f}^{(0)}\left(\xi_{1}-\xi_{2}\right)=\left\langle\psi_{ \pm}\left(\xi_{1}\right) \psi_{ \pm}^{*}\left(\xi_{2}\right)\right\rangle_{f}^{(0)}=\operatorname{sgn}\left(t_{1}-t_{2}\right) \frac{i}{2 \pi} \frac{1}{ \pm x+i t}\left(\frac{\delta^{2}}{x^{2}+t^{2}}\right)^{\Delta}
$$

where $\Delta=\frac{1}{4}\left(\frac{\pi}{\beta^{2}}+\frac{\beta^{2}}{\pi}-2\right)$ and $\xi=(x, t)$. The appearance of the factor $\operatorname{sgn}\left(t_{1}-t_{2}\right)$ is in accordance with the formula (24). Indeed, due to this formula the fermion Green function with proper time-ordering is 


$$
G_{ \pm}^{(0)}\left(\xi_{1}-\xi_{2}\right)=\left\langle\hat{T}_{t} \hat{\psi}_{ \pm}\left(x_{1}, t_{1}\right) \hat{\psi}_{ \pm}^{\dagger}\left(x_{2}, t_{2}\right)\right\rangle_{H}^{(0)}=\frac{i}{2 \pi} \frac{1}{ \pm x+i t}\left(\frac{\delta^{2}}{x^{2}+t^{2}}\right)^{\Delta}
$$

which is the right answer.

It will be convenient for us to introduce the following generating functional

$$
Z_{ \pm}\left(f_{0}, f_{1}\right)=\left\langle\psi_{ \pm}\left(\xi_{1}\right) \psi_{ \pm}^{*}\left(\xi_{2}\right) e^{\int d^{2} \xi\left[f_{0}(\xi) \Pi(\xi)+f_{1}(\xi) \partial_{1} \Phi(\xi)\right]}\right\rangle_{f}^{(0)}
$$

This functional can be calculated using the formula (33)

$$
Z_{ \pm}\left(f_{0}, f_{1}\right)=G_{ \pm, f}^{(0)}\left(\xi_{1}, \xi_{2}\right) e^{F^{ \pm}\left(f_{0}, f_{1}\right)+\frac{1}{2} \int d^{2} \xi_{1} d^{2} \xi_{2} f^{T}\left(\xi_{1}\right) D\left(\xi_{1}, \xi_{2}\right) f\left(\xi_{2}\right)}
$$

In this formula $G_{ \pm, f}^{(0)}$ is the Green function (34), $f=\left(f_{0}, f_{1}\right)^{T}$ is a two-vector constructed from $f_{0}, f_{1}$, and $F^{ \pm}\left(f_{0}, f_{1}\right)$ is a linear functional of $f_{0}, f_{1}$

$$
F^{ \pm}\left(f_{0}, f_{1}\right)=\int d^{2} \xi\left(f_{0}(\xi) J_{0}^{ \pm}\left(\xi_{1}, \xi_{2}, \xi\right)+f_{1}(\xi) J_{1}^{ \pm}\left(\xi_{1}, \xi_{2}, \xi\right)\right)
$$

where

$$
\begin{aligned}
& J_{0}^{ \pm}\left(\xi_{1}, \xi_{2}, \xi_{3}\right)=\frac{1}{2 \pi} \frac{-i \frac{\pi}{\beta}\left(x_{1}-x_{3}\right) \mp \beta\left(t_{1}-t_{3}\right)}{\left(t_{1}-t_{3}\right)^{2}+\left(x_{1}-x_{3}\right)^{2}}-\left(x_{1}, t_{1} \leftrightarrow x_{2}, t_{2}\right), \\
& J_{1}^{ \pm}\left(\xi_{1}, \xi_{2}, \xi_{3}\right)=\frac{1}{2 \pi} \frac{ \pm i \beta\left(x_{1}-x_{3}\right)+\frac{\pi}{\beta}\left(t_{1}-t_{3}\right)}{\left(t_{1}-t_{3}\right)^{2}+\left(x_{1}-x_{3}\right)^{2}}-\left(x_{1}, t_{1} \leftrightarrow x_{2}, t_{2}\right) .
\end{aligned}
$$

Finally, $D$ is a matrix Green function which in the momentum space is

$$
D(\omega, p)=\frac{p^{2}}{p^{2}+\omega^{2}}\left[\begin{array}{cc}
1 & i \frac{\omega}{p} \\
i \frac{\omega}{p} & 1
\end{array}\right] .
$$

So far we have calculated the generating functional which corresponds to the free-boson Lagrangian $L_{\mathcal{E}}^{(0)}$. But the averaging in (17) is done with respect to the Lagrangian $L_{\mathcal{E}}^{(1)}(25)$, and therefore we need the following generating functional

$$
Z^{-1} \int D \Pi D \Phi \psi_{ \pm}\left(\xi_{1}\right) \psi_{ \pm}^{*}\left(\xi_{2}\right) e^{L_{\mathcal{E}}^{(1)}+\int d^{2} \xi\left[f_{0}(\xi) \Pi(\xi)+f_{1}(\xi) \partial_{1} \Phi(\xi)\right]}
$$

where

$$
Z=\int D \Pi D \Phi e^{L_{\mathcal{E}}^{(1)}}
$$


Before doing the perturbation theory in $V$ it is convenient to shift the fields $\Pi \rightarrow \Pi+$ $J_{0}^{ \pm}, \partial_{1} \Phi \rightarrow \partial_{1} \Phi+J_{1}^{ \pm}$and redefine them $\Pi=\gamma_{0}, \partial_{1} \Phi=\gamma_{1}:$

$$
\begin{array}{r}
Z^{-1} \int D \Pi D \Phi \psi_{ \pm}\left(\xi_{1}\right) \psi_{ \pm}^{*}\left(\xi_{2}\right) e^{L_{\mathcal{E}}^{(1)}+\int d^{2} \xi\left(f_{0} \Pi+f_{1} \partial_{1} \Phi\right)} \\
=G_{ \pm, f}^{(0)}\left(\xi_{1}-\xi_{2}\right) Z_{\gamma}^{-1} \int D \gamma_{0} D \gamma_{1} e^{\int d^{2} \xi\left[-\frac{1}{2} \gamma^{T} \hat{D}^{-1} \gamma+V\left(\gamma_{0}+J_{0}, \gamma_{1}+J_{1}\right)+f_{0}\left(\gamma_{0}+J_{0}\right)+f_{1}\left(\gamma_{1}+J_{1}\right)\right]}
\end{array}
$$

In this representation $V$ is the interaction term

$$
V\left(\gamma_{0}, \gamma_{1}\right)=-\frac{\alpha}{3} \beta \gamma_{1}\left(\frac{3 \pi}{\beta^{2}} \gamma_{0}^{2}+\frac{\beta^{2}}{\pi} \gamma_{1}^{2}\right)
$$

and

$$
Z_{\gamma}=\int D \gamma_{0} D \gamma_{1} e^{\int d^{2} \xi\left[-\frac{1}{2} \gamma^{T} \hat{D}^{-1} \gamma+V\left(\gamma_{0}, \gamma_{1}\right)\right]}
$$

The arguments of the $J$-functions in (42) are $J\left(\xi_{1}, \xi_{2}, \xi\right)$. The field $\gamma$ is a two-component field $\gamma=\left(\gamma_{0}, \gamma_{1}\right)^{T}$ and $\hat{D}^{-1}$ is an integral operator corresponding to the Green function $D^{-1}$, which in the momentum space is the inverse Green function (41)

$$
D^{-1}(\omega, p)=\left[\begin{array}{cc}
1 & -i \frac{\omega}{p} \\
-i \frac{\omega}{p} & 1
\end{array}\right] .
$$

This representation is convenient for the perturbation theory in $\alpha$ because it already contains the Green function $G_{ \pm}^{(0)}$ so that we do not have to deal with $\psi$-functionals any more. To prove the representation (42) it is convenient to use the following formulas for $J$-functions:

$$
\begin{array}{r}
J_{0}^{ \pm}\left(\xi_{1}, \xi_{2}, \xi\right)=\left[i \frac{\pi}{\beta} \frac{\partial}{\partial x} \pm \beta \frac{\partial}{\partial t}\right]\left[d\left(\xi_{1}-\xi\right)-d\left(\xi_{2}-\xi\right)\right], \\
J_{1}^{ \pm}\left(\xi_{1}, \xi_{2}, \xi\right)=\left[\mp \beta i \frac{\partial}{\partial x}-\frac{\pi}{\beta} \frac{\partial}{\partial t}\right]\left[d\left(\xi_{1}-\xi\right)-d\left(\xi_{2}-\xi\right)\right], \\
{\left[\partial_{0}^{2}+\partial_{1}^{2}\right] d(\xi)=\delta(\xi)}
\end{array}
$$

where $d(\xi)$ is defined in (31).

\section{CALCULATION OF THE HALL CONDUCTIVITY.}

To calculate $\Gamma$ we need to find the correlators

$$
K_{ \pm}\left(\xi_{1}, \xi_{2}, \xi_{3}\right)=\int d x_{3}\left\langle j\left(\xi_{3}\right) \psi_{ \pm}\left(\xi_{1}\right) \psi_{ \pm}^{\dagger}\left(\xi_{2}\right)\right\rangle
$$


and

$$
G_{ \pm}(\xi)=\left\langle\psi(\xi) \psi^{\dagger}(0)\right\rangle
$$

where the averaging is done with respect the single-chain Lagrangian (15). Let us apply the technique developed in the previous section to calculate these correlators. First of all we should find these correlators to the zeroth order in $\alpha$. The Green function $G_{ \pm}^{(0)}$ was already calculated in the previous section. The correlator $K_{ \pm}$to the zeroth order in $\alpha$ can be calculated using the generating functional (36, 37)

$$
\begin{aligned}
K_{ \pm}^{(0)}= & -\frac{e v_{F}}{\beta} \operatorname{sgn}\left(t_{1}-t_{2}\right) \int d x_{3} \frac{\delta}{\delta f_{0}} Z_{ \pm}\left(f_{0}, f_{1}\right)_{\mid f_{0}=f_{1}=0} \\
& =-\frac{e v_{F}}{\beta} \int d x_{3} G_{ \pm}^{(0)}\left(\xi_{1}, \xi_{2}\right) J_{0}\left(\xi_{1}, \xi_{2}, \xi_{3}\right) .
\end{aligned}
$$

Calculating the integral over $x_{3}$ we get

$$
\begin{array}{r}
K_{ \pm}^{(0)}=\int d x_{3}\left\langle j_{0}\left(\xi_{3}\right) \psi_{ \pm}\left(\xi_{1}\right) \psi_{ \pm}^{\dagger}\left(\xi_{2}\right)\right\rangle^{(0)} \\
=\mp \frac{1}{2} e v_{F} G_{ \pm}^{(0)}\left(\xi_{1}-\xi_{2}\right)\left[\operatorname{sgn}\left(t_{3}-t_{1}\right)-\operatorname{sgn}\left(t_{3}-t_{2}\right)\right] .
\end{array}
$$

Substituting these expressions and the Green function (35) into $\Gamma$ (17) we get zero due to the fact that $G_{ \pm}^{(0)}\left(x_{1}-x_{2}, t_{1}-t_{2}\right)$ is odd under the transformation $x_{1}, t_{1} \leftrightarrow x_{2}, t_{2}$ and $K_{ \pm}^{(0)}$ is even. Indeed, the model with the linear spectrum $(\alpha=0)$ should give zero Hall effect due to the particle-hole symmetry. To the first order in $\alpha$ we can conveniently represent the product of these two correlators as

$$
\left\langle j \psi \psi^{\dagger}\right\rangle\left\langle\psi \psi^{\dagger}\right\rangle=\left\langle j_{0} \psi \psi^{\dagger}\right\rangle\left\langle\psi \psi^{\dagger}\right\rangle+\left\langle j_{1} \psi \psi^{\dagger}\right\rangle^{(0)}\left\langle\psi \psi^{\dagger}\right\rangle^{(0)}
$$

where $j_{0}$ and $j_{1}$ are defined in (12). The first term in (47) contains the current to the zeroth order so that the nonlinear corrections come from the Lagrangian. The second term contains no $\alpha$-corrections from the Lagrangian because $j_{1}$ is already proportional to $\alpha$. Fortunately the first term gives no contribution when substituted into the formula for $\Gamma$ (see Appendix B) and therefore we should calculate only the contribution from the second term. The correlator

$$
\int d x_{3}\left\langle j_{1}\left(\xi_{3}\right) \psi_{ \pm}\left(\xi_{1}\right) \psi_{ \pm}\left(\xi_{2}\right)\right\rangle^{(0)}
$$


where the current correction $j_{1}$ in the bosonized form is

$$
j_{1}=-2 e \alpha \Pi \partial_{1} \Phi
$$

can be calculated using the formulas (36,37)

$$
\begin{gathered}
\int d x_{3}\left\langle j_{1}\left(\xi_{3}\right) \psi_{ \pm}\left(\xi_{1}\right) \psi_{ \pm}\left(\xi_{2}\right)\right\rangle^{(0)} \\
=-2 e \alpha \int d x_{3} J_{0}^{ \pm}\left(\xi_{1}, \xi_{2}, \xi_{3}\right) J_{1}^{ \pm}\left(\xi_{1}, \xi_{2}, \xi_{3}\right) G_{ \pm}^{(0)}\left(\xi_{1}, \xi_{2}\right),
\end{gathered}
$$

where it was used that $\int d x_{1} D\left(\xi_{1}, \xi_{2}\right)=0$ ( see Appendix B). Taking the integral over $x_{3}$ we get

$$
\begin{gathered}
\int d x_{3}\left\langle j_{1}\left(\xi_{3}\right) \psi_{ \pm}\left(\xi_{1}\right) \psi_{ \pm}^{\dagger}\left(\xi_{2}\right)\right\rangle=\frac{\alpha e i}{2 \pi} G_{ \pm}^{(0)}\left(\xi_{1}-\xi_{2}\right) \frac{1}{\left(x_{1}-x_{2}\right)^{2}+\left(t_{1}-t_{2}\right)^{2}} \\
{\left[ \pm 2 \pi i\left(t_{1}-t_{2}\right)-\left((\pi / \beta)^{2}+\beta^{2}\right)\left(x_{1}-x_{2}\right)\right]\left[\operatorname{sgn}\left(t_{3}-t_{1}\right)-\operatorname{sgn}\left(t_{3}-t_{2}\right)\right]}
\end{gathered}
$$

Substituting (48) and (35) into the expression for $\Gamma$ and shifting the variables $x_{2} \rightarrow x_{2}+$ $x_{1}, t_{2} \rightarrow t_{2}+t_{1}$ for $\Gamma_{\mathcal{E}}(\omega)$ we get

$$
\Gamma_{\mathcal{E}}(\omega)=\frac{e \alpha i}{2 \pi^{3}} \delta^{4 \Delta} \int d t^{\prime} \int d t e^{i \omega t}\left[\operatorname{sgn} t-\operatorname{sgn}\left(t^{\prime}+t\right)\right] f\left(t^{\prime}\right)
$$

where

$$
f(t)=\int d x \frac{1}{(x+i t)^{2}} \frac{1}{\left(x^{2}+t^{2}\right)^{1+2 \Delta}}\left[-2 \pi i t+x\left((\pi / \beta)^{2}+\beta^{2}\right)\right] \sin q x .
$$

Using that $f\left(t^{\prime}\right)$ is an even function of $t^{\prime}$ and integrating by parts one can show that

$$
\begin{gathered}
\int d t^{\prime} \int d t e^{i \omega t}\left[\operatorname{sgn} t-\operatorname{sgn}\left(t+t^{\prime}\right)\right] f\left(t^{\prime}\right) \\
=-\frac{4 i}{\omega} \int_{0}^{\infty} d t(\cos \omega t-1) f(t) .
\end{gathered}
$$

Therefore

$$
\Gamma_{\mathcal{E}}(\omega)=\frac{2 e \alpha}{\omega \pi^{3}} \delta^{4 \Delta} \int_{0}^{\infty} d t(\cos \omega t-1) f(t)
$$

Introducing cylindrical coordinates $x_{2}=\rho \cos \phi, t=\rho \sin \phi$ we can integrate over $\phi$ obtaining 


$$
\begin{array}{r}
\Gamma_{\mathcal{E}}(\omega)=\frac{e \alpha}{\pi^{2} \omega} \delta^{4 \Delta} \int_{0}^{\infty} \frac{d \rho}{\rho^{2(1+2 \Delta)}}\left[(\pi / \beta-\beta)^{2} \frac{q}{\sqrt{\omega^{2}+q^{2}}} J_{1}\left(\rho \sqrt{\omega^{2}+q^{2}}\right)\right. \\
\left.-(\pi / \beta+\beta)^{2} \frac{q\left(q^{2}-3 \omega^{2}\right)}{\left(\omega^{2}+q^{2}\right)^{3 / 2}} J_{3}\left(\rho \sqrt{\omega^{2}+q^{2}}\right)-(\omega=0)\right]
\end{array}
$$

where $J_{1}$ and $J_{3}$ are the Bessel functions (not to be confused with $J$-functions (39,40)). Calculating the integral over $\rho$ and using (16) for the Hall conductivity we get

$$
\sigma_{\perp}(\omega)=-\frac{2 e^{2} t_{\perp}^{2} \alpha}{\pi}\left(\frac{\delta}{2}\right)^{4 \Delta} \frac{\Gamma(1-2 \Delta)}{\Gamma(2+2 \Delta)} \frac{q}{\omega^{2}}\left[\left(q^{2}-\omega^{2}\right)^{2 \Delta} \frac{q^{2}+\omega^{2}}{q^{2}-\omega^{2}}-q^{4 \Delta}\right],
$$

which is the main result of this paper. Expanding in $\omega$ we get the DC-conductivity

$$
\sigma_{\perp}=-\frac{4}{\pi} \frac{\Gamma(1-2 \Delta)}{\Gamma(2+2 \Delta)}(1-\Delta) \frac{t_{\perp}^{2} e^{2} \alpha}{v_{B}^{2}\left(\frac{e H a_{y} v_{B}}{c}\right)^{1-4 \Delta}}\left(\frac{\delta}{2 v_{B}}\right)^{4 \Delta},
$$

where $v_{B}$ was restored. One can see that $\sigma_{\perp}$ depends on the magnetic field as $H^{4 \Delta-1}$. Note that the formula (53) does not work for $\Delta>\frac{1}{2}$. (The integral over $\rho$ in (51) does not converge in this case.) The fact that $\Delta=\frac{1}{2}$ is a critical value is not surprising because it was shown 3 that in the case $\Delta>\frac{1}{2}$ the interchain hopping term in the Hamiltonian is irrelevant in RG sense. To avoid confusion we note that the irrelevance of the hopping term in the case $\Delta>\frac{1}{2}$ should be understood in the straightforward dimensional RG sense. Actually, considering two-particle tunneling processes, one can see that the hopping term is relevant even for $\Delta>\frac{1}{2}$ (See 10 ). As was mentioned above, this theory is valid for high magnetic fields. To find the applicability criterion we should consider the next nonzero order in $t_{\perp}$. It is hard to calculate it but from dimensional analysis it follows that the correction should have the form

$$
\sigma_{\perp} \rightarrow \sigma_{\perp}\left(1+\text { const } \cdot \frac{t_{\perp}^{2}}{\left(\frac{e H a_{y} v_{F}}{c}\right)^{2}}\left(\frac{e H a_{y} \delta}{c}\right)^{4 \Delta}\right)
$$

Indeed, expanding to the next nonzero order in $t_{\perp}$ one gets four additional $\psi$-operators which give the factor $\delta^{4 \Delta}$ and the remaining factors can be restored from dimensionality. This correction is small if

$$
H \gg \frac{\Phi_{0}}{a_{x} a_{y}}\left(\frac{t_{\perp}}{\epsilon_{F}}\right)^{\frac{1}{1-2 \Delta}},
$$

where $\Phi_{0}$ is a quantum of magnetic flux. It agrees with our expectation (11) in the Introduction. 


\section{DISCUSSION AND CONCLUSIONS.}

We considered the Hall effect in a quasi-one-dimensional interacting electron system. It was found that in high magnetic fields

$$
H \gg \frac{\Phi_{0}}{a_{x} a_{y}}\left(\frac{t_{\perp}}{\epsilon_{F}}\right)^{\frac{1}{1-2 \Delta}}
$$

there is a power law dependence of the Hall conductivity on the magnetic field

$$
\sigma_{\perp} \sim H^{-1+4 \Delta}
$$

where $2 \Delta$ is the anomalous exponent of the one-dimensional Green function (35). This formula can be applied for $\Delta<\frac{1}{2}$. This result was obtained for the zero-temperature

case. But for nonzero temperatures much lower than $\frac{e H a_{x} v_{F}}{c}$ it should still hold because the temperature will change the correlation functions only in the low-energy region which is irrelevant in the case of high magnetic fields. Therefore this result can be applied for nonzero temperatures if

$$
\frac{T}{\epsilon_{F}} \ll \frac{H a_{x} a_{y}}{\Phi_{0}} .
$$

We considered the simplest model of spinless electrons. In a more realistic case of the Hubbard model the single-chain Green function has a more complicated form

$$
G(x, t)=\frac{i}{2 \pi} \frac{1}{\left( \pm x+v_{\rho} i t\right)^{1 / 2}\left( \pm x+v_{s} i t\right)^{1 / 2}}\left(\frac{\delta^{2}}{x^{2}+\left(v_{\rho} t\right)^{2}}\right)^{\Delta}
$$

(in the Euclidean space), where $v_{s}$ and $v_{\rho}$ are the spin and charge velocities. Using dimensional analysis one can argue that the result (55) should still hold. Indeed, the formula (11) is right in this case. Comparing this formula with the final answer, one can see that every $\psi$-operator gives rise to an additional factor $\delta^{\Delta}$ in the final answer, while the current $j$ gives no additional powers because $j$ is a local function of the Bose-fields. Therefore the exponent of the magnetic field in the final answer should be the same with $\Delta$ defined by (57).

We hope that experimentally this effect can be observed in one-dimensional organic conductors (see b $_{\text {for }}$ review). Actually, the possibility of observing this effect depends on 
the value of $\Delta$ in a particular material. If we take $a_{x} a_{y}=5 \times 10^{-15} \mathrm{~cm}^{2}$ and $\frac{t_{\perp}}{\epsilon_{F}}=\frac{1}{10}$ which is typical for these materials, then we can rewrite the condition (54) in the form

$$
H \gg\left(10^{3} \ldots 10^{4}\right)\left(\frac{1}{10}\right)^{\frac{1}{1-2 \Delta}} T .
$$

It can be satisfied from the experimental point of view if $\Delta$ is not far from $\frac{1}{2}$. Note that one-dimensional organic conductors at low temperatures typically exhibit phase transitions to superconducting, CDW or SDW states and many properties such as Hall effect, magnetoresistance, etc. are very unusual at these temperatures. Of course our result can not be applied in these cases because effects of complicated ground state were not considered. In other words, for this effect to be observed the materials must be in the metallic state.

\section{ACKNOWLEDGMENTS}

I am indebted to L. B. Ioffe for the idea of this work and very useful discussions.

\section{APPENDIX A. BOSONIZATION OF THE SINGLE-CHAIN HAMILTONIAN.}

In this section we will follow the approach described int. In the bosonization technique the product of two operators which has an infinite vacuum matrix element

$$
\langle\hat{A} \hat{B}\rangle \rightarrow \infty
$$

should be understood as a limit

$$
\langle\hat{A}(x) \hat{B}(x)\rangle \rightarrow\left\langle\hat{A}(x) \hat{B}\left(x^{\prime}\right)\right\rangle_{x \rightarrow x^{\prime}}
$$

Usually the r.h.s. of this expression can be written as

$$
\frac{z}{\left(x-x^{\prime}\right)^{\eta}}+\hat{C}(x)+\ldots
$$

where $z, \eta$ are some constants. The first term is divergent but it is a c-number and it can be ignored if we are interested in operators in normal ordered sense. The second term is well 
defined and the others are equal to zero in the limit $x \rightarrow x^{\prime}$. Our task is to bosonize the single-chain Hamiltonian

$$
H_{1}=\int d x\left(-i v_{F} \hat{\psi}^{\dagger} \tau_{3} \partial_{1} \hat{\psi}-\alpha \hat{\psi}^{\dagger} \partial_{1}^{2} \hat{\psi}+g \hat{\psi}_{+}^{\dagger} \hat{\psi}_{+} \hat{\psi}_{-}^{\dagger} \hat{\psi}_{-}\right)
$$

Using the Baker-Hausdorff relation

$$
e^{A} e^{B}=e^{A+B} e^{\frac{1}{2}[A, B]}
$$

we can write the product of two $\psi$-operators as

$$
\hat{\psi}_{ \pm}^{\dagger}(x) \hat{\psi}_{ \pm}\left(x_{1}\right)= \pm \frac{1}{2 \pi i\left(x_{1}-x\right)}: e^{\mp i\left(\hat{\Phi}_{ \pm}(x)-\hat{\Phi}_{ \pm}\left(x_{1}\right)\right)}:
$$

where

$$
\begin{gathered}
\hat{\psi}_{ \pm}(x, t)=\frac{1}{\sqrt{2 \pi \delta}} e^{ \pm i \hat{\Phi}_{ \pm}(x, t)} \\
\hat{\Phi}_{ \pm}(x, t)=\sqrt{\pi}\left(\hat{\Phi}(x, t) \mp \int_{-\infty}^{x} \hat{\Pi}\left(x^{\prime}, t\right) d x^{\prime}\right),
\end{gathered}
$$

and : : means normal ordering of operators. Here we have rescaled the energy units so that $v_{B}=1$. Expanding the exponent to the third order in $\left(x_{1}-x\right)$, we get

$$
\begin{array}{r}
\hat{\psi}_{ \pm}^{\dagger}(x) \hat{\psi}_{ \pm}\left(x_{1}\right)=: \pm \frac{1}{2 \pi i}\left[ \pm i \hat{\Phi}_{ \pm}^{\prime}(x)-\frac{1}{2}\left(x_{1}-x\right)\left(\left(\hat{\Phi}_{ \pm}^{\prime}(x)\right)^{2} \mp i \hat{\Phi}_{ \pm}^{\prime \prime}(x)\right)\right. \\
\left.+\frac{1}{6}\left(x_{1}-x\right)^{2}\left( \pm i \hat{\Phi}_{ \pm}^{\prime \prime \prime}(x)-3 \Phi_{ \pm}^{\prime}(x) \Phi_{ \pm}^{\prime \prime}(x) \mp i\left(\hat{\Phi}_{ \pm}^{\prime}(x)\right)^{3}\right)\right]
\end{array}
$$

where all singular c-number terms are ignored and the primes denote differentiation with respect to $x$. Now it is straightforward to bosonize all operators which we need. For the terms of the Hamiltonian we have

$$
\begin{gathered}
-i v_{F} \int d x \hat{\psi}^{\dagger} \tau_{3} \partial_{1} \hat{\psi}=\frac{1}{2} \int d x\left(v_{B}\left(\partial_{1} \Phi\right)^{2}+\frac{1}{v_{B}} \Pi^{2}\right) v_{F} \\
g \int d x \hat{\psi}_{+}^{\dagger} \hat{\psi}_{+} \hat{\psi}_{-}^{\dagger} \hat{\psi}_{-}=\frac{g}{4 \pi} \int d x\left(v_{B}\left(\partial_{1} \Phi\right)^{2}-\frac{1}{v_{B}} \Pi^{2}\right) \\
\int d x \hat{\psi}^{\dagger} \partial_{1}^{2} \hat{\psi}=-\frac{1}{3} \frac{\pi}{\sqrt{v_{B}}} \int d x \partial_{1} \Phi\left(3 \Pi^{2}+v_{B}^{2}\left(\partial_{1} \Phi\right)^{2}\right)
\end{gathered}
$$

where $v_{B}$ was restored for a reason which will be seen below. Normal ordering of operators is implied hereafter. In the derivation of the above expressions the terms in (58) which are 
total derivatives were neglected. The sum of the first and the second terms can be written as the usual free-boson Hamiltonian if we renormalize the fields $\Phi \rightarrow \frac{\beta}{\sqrt{\pi}} \Phi, \Pi \rightarrow \frac{\sqrt{\pi}}{\beta} \Pi$ so that

$$
-i v_{F} \int d x \hat{\psi}^{\dagger} \tau_{3} \partial_{1} \hat{\psi}+g \int d x \hat{\psi}_{+}^{\dagger} \hat{\psi}_{+} \hat{\psi}_{-}^{\dagger} \hat{\psi}_{-}=\int d x \frac{1}{2}\left(v_{B}^{2}\left(\partial_{1} \Phi\right)^{2}+\Pi^{2}\right)
$$

where

$$
\begin{gathered}
\beta^{4}=\pi^{2} \frac{v_{F}-\frac{g}{2 \pi}}{v_{F}+\frac{g}{2 \pi}}, \\
v_{B}=\sqrt{v_{F}^{2}-\left(\frac{g}{2 \pi}\right)^{2}} .
\end{gathered}
$$

Knowing the relation between $v_{B}$ and $v_{F}$ we can put $v_{B}=1$ again. Note that the above relations depend on a cut-of procedure and are universal only to the first power in $g$. So the single-chain Hamiltonian expressed through the new renormalized fields becomes

$$
\hat{H}_{1}=\frac{1}{2}\left(\left(\partial_{1} \hat{\Phi}\right)^{2}+\hat{\Pi}^{2}\right)+\frac{\alpha}{3} \beta \partial_{1} \hat{\Phi}\left(3 \frac{\pi}{\beta^{2}} \hat{\Pi}^{2}+\frac{\beta^{2}}{\pi}\left(\partial_{1} \hat{\Phi}\right)^{2}\right)
$$

Finally, the current operator expressed through the same fields is

$$
\hat{j}=-e\left(\frac{v_{F}}{\beta} \hat{\Pi}+2 \alpha \hat{\Pi} \partial_{1} \hat{\Phi}\right)
$$

\section{APPENDIX B}

Lel us prove that to the first order in $\alpha$ there is no contribution to $\Gamma$ from the first term in $(47)$

$$
\left\langle j_{0}\left(\xi_{3}\right) \psi_{ \pm}\left(\xi_{1}\right) \psi_{ \pm}^{*}\left(\xi_{2}\right)\right\rangle\left\langle\psi_{ \pm}\left(\xi_{2}\right) \psi_{ \pm}^{*}\left(\xi_{1}\right)\right\rangle
$$

To the zeroth order in $\alpha$ it was already proven that this term gives no contribution to Г. Therefore we should show that the first order corrections give no contribution as well. According to (42), to the first order in $\alpha$ we have

$$
\left\langle\psi_{ \pm}\left(\xi_{1}\right) \psi_{ \pm}^{*}\left(\xi_{2}\right)\right\rangle=G_{ \pm}^{(0)}\left(\xi_{1}-\xi_{2}\right)\left(1+\left\langle\int d^{2} \xi V\left(\gamma_{0}+J_{0}^{ \pm}, \gamma_{1}+J_{1}^{ \pm}\right)_{\xi}\right\rangle_{\gamma}\right)
$$


where the subscript $\xi$ represents the arguments of the $\gamma$-fields and $J$-functions, for example

$$
V\left(\gamma_{0}+J_{0}^{ \pm}, \gamma_{1}+J_{1}^{ \pm}\right)_{\xi} \equiv V\left(\gamma_{0}(\xi)+J_{0}^{ \pm}\left(\xi_{1}, \xi_{2}, \xi\right), \gamma_{1}(\xi)+J_{1}^{ \pm}\left(\xi_{1}, \xi_{2}, \xi\right)\right)
$$

and the symbol \langle\rangle$_{\gamma}$ means averaging with the functional

$$
e^{-\frac{1}{2} \int d^{2} \xi \gamma^{T} \hat{D}^{-1} \gamma}
$$

It was also used that $\left\langle V\left(\gamma_{0}, \gamma_{1}\right)\right\rangle_{\gamma}=0$ to get (61). For the correlator containing $j_{0}$, using the same formula to the first order in $\alpha$ we get

$$
\begin{array}{r}
\left\langle j_{0}\left(\xi_{3}\right) \psi_{ \pm}\left(\xi_{1}\right) \psi_{ \pm}^{*}\left(\xi_{2}\right)\right\rangle \\
=-\frac{e v_{F}}{\beta} G_{ \pm}^{(0)}\left(\xi_{1}-\xi_{2}\right)\left\langle\left[1+\int d \xi V\left(\gamma_{0}+J_{0}^{ \pm}, \gamma_{1}+J_{1}^{ \pm}\right)_{\xi}\right]\left(\gamma_{0}+J_{0}^{ \pm}\right)_{\xi_{3}}\right\rangle_{\gamma} \\
=-\frac{e v_{F}}{\beta} G_{ \pm}^{(0)}\left(\xi_{1}-\xi_{2}\right) J_{0}^{ \pm}\left(\xi_{1}, \xi_{2}, \xi_{3}\right)\left(1+\left\langle\int d \xi V\left(\gamma_{0}+J_{0}^{ \pm}, \gamma_{1}+J_{1}^{ \pm}\right)_{\xi}\right\rangle_{\gamma}\right) \\
-\frac{e v_{F}}{\beta} G_{ \pm}^{(0)}\left(\xi_{1}-\xi_{2}\right)\left\langle\int d \xi V\left(\gamma_{0}+J_{0}^{ \pm}, \gamma_{1}+J_{1}^{ \pm}\right)_{\xi} \gamma_{0}\left(\xi_{3}\right)\right\rangle_{\gamma},
\end{array}
$$

where the arguments of $J_{0}, J_{1}$ are implied according to the same rule as above. Note that the functions $J_{0}, J_{1}$ are odd under the exchange of the arguments $\xi_{1}$ and $\xi_{2}$. Therefore the term

$$
\int d \xi\left\langle V\left(\gamma_{0}+J_{0}^{ \pm}, \gamma_{1}+J_{1}^{ \pm}\right)_{\xi}\right\rangle_{\gamma} \equiv \int d \xi\left\langle V\left(\gamma_{0}(\xi)+J_{0}^{ \pm}\left(\xi_{1}, \xi_{2}, \xi\right), \gamma_{1}(\xi)+J_{1}^{ \pm}\left(\xi_{1}, \xi_{2}, \xi\right)\right)\right\rangle_{\gamma}
$$

is odd under this exchange too. (One can always change $\gamma_{0}, \gamma_{1} \rightarrow-\gamma_{0},-\gamma_{1}$ because the functional (62) is invariant under this transformation.) One can see that after the substitution of $(61,63)$ into $(60)$ the correction in $(61)$ and the correction of the same kind in (63) cancel each other (to the first order) and (60) becomes

$$
\begin{array}{r}
\left\langle j_{0}\left(\xi_{3}\right) \psi_{ \pm}\left(\xi_{1}\right) \psi_{ \pm}^{*}\left(\xi_{2}\right)\right\rangle_{\gamma}\left\langle\psi_{ \pm}\left(\xi_{2}\right) \psi_{ \pm}^{*}\left(\xi_{1}\right)\right\rangle_{\gamma}=-\frac{e v_{F}}{\beta} G_{ \pm}^{(0)}\left(\xi_{1}-\xi_{2}\right) G_{ \pm}^{(0)}\left(\xi_{2}-\xi_{1}\right) J_{0}^{ \pm}\left(\xi_{1}, \xi_{2}, \xi_{3}\right) \\
-\frac{e v_{F}}{\beta} G_{ \pm}^{0}\left(\xi_{1}-\xi_{2}\right) G_{ \pm}^{0}\left(\xi_{2}-\xi_{1}\right)\left\langle\int d \xi V\left(\gamma_{0}+J_{0}^{ \pm}, \gamma_{1}+J_{1}^{ \pm}\right)_{\xi} \gamma_{0}\left(\xi_{3}\right)\right\rangle_{\gamma}
\end{array}
$$

The first term in this expression is the zero order term which gives no contribution to $\Gamma$. The second term gives zero when integrated over $x_{3}$. To see this we need the correlation 
functions of the $\gamma$-fields. Taking the Fourier transformation of the Green function (41) one gets

$$
\begin{gathered}
\left\langle\gamma_{1}\left(\xi_{1}\right) \gamma_{1}\left(\xi_{2}\right)\right\rangle_{\gamma}=\left\langle\gamma_{0}\left(\xi_{1}\right) \gamma_{0}\left(\xi_{2}\right)\right\rangle_{\gamma}=\frac{1}{4 \pi}\left(\frac{1}{(t+i x)^{2}}+\frac{1}{(t-i x)^{2}}\right) \\
\left\langle\gamma_{1}\left(\xi_{1}\right) \gamma_{0}\left(\xi_{2}\right)\right\rangle_{\gamma}=\left\langle\gamma_{0}\left(\xi_{1}\right) \gamma_{1}\left(\xi_{2}\right)\right\rangle_{\gamma}=-\frac{1}{4 \pi}\left(\frac{1}{(t+i x)^{2}}-\frac{1}{(t-i x)^{2}}\right) .
\end{gathered}
$$

From the form of the $\gamma$-correlators written above one can see that

$$
\int d x_{1}\left\langle\gamma\left(\xi_{1}\right) \gamma^{\prime}\left(\xi_{2}\right)\right\rangle=0
$$

where $\gamma$ and $\gamma^{\prime}$ are any fields from the $\gamma_{1}, \gamma_{2}$-fields. Therefore there is no contribution to $\Gamma$ from the term $(60)$. 


\section{REFERENCES}

${ }^{1}$ R. Shankar, Low-Dimensional Quantum Field Theories for Condensed Matter Physicists ( World Scientific Publishing Co. Pte. Ltd., 1992), p. 353

${ }^{2}$ E. Fradkin, Field Theories of Condensed Matter Systems (Addison-Wesley,Redwood City, 1991)

${ }^{3}$ X.G. Wen, Phys. Rev. B 42, 6623 (1990).

${ }^{4}$ F.D.M. Haldane, J. Phys. C 14, 2585 (1981).

${ }^{5}$ H.J. Schulz, Int.J.Mod.Phys. B 5, 57 (1991).

${ }^{6}$ D. Jerome and H.J.Schulz, Adv. Phys. 31, 299 (1982).

${ }^{7}$ M.E. Peskin and D.V. Schroeder, An Introduction to Quantum Field Theory (AddisonWesley Publishing Company, 1995).

${ }^{8}$ S. Brazovskii and V.Yakovenko, Sov. Phys. JETP 62, 1340 (1985)

${ }^{9}$ D.Boies, C.Bourbonnais and A.-M.S.Tremblay, Phys. Rev. Lett. 74, 968 (1995)

${ }^{10}$ V.M. Yakovenko, JETP Lett. 56, 510 (1992)

${ }^{11}$ L.P. Gor'kov, Sov. Phys. Usp. 27, 809 (1984) 